\title{
DECOHERENCE-FREE SUBSPACES FOR OPEN QUANTUM RANDOM WALKS ON GRAPHS
}

\author{
J. Agredo \\ Department of Mathematics \\ National University of Colombia \\ Department of Mathematics \\ Colombian School of Engineering Julio Garavito \\ Bogotá, COLOMBIA
}

\begin{abstract}
We study decoherence-free subspaces in a type of Quantum Markov Semigroups called continuous-time open quantum random walks on graphs. We measure the temporary changes of quantum correlations using geometric quantum discord with bures distance under some assumptions about the semigroup. In particular, we characterize the decay of correlations to zero, showing that turns out to be closely related with the structure of decoherence-free subspace.
\end{abstract}

AMS Subject Classification: 47D06, 46L55, 81S22

Key Words: quantum Markov semigroup, geometric quantum discord, Bures distance, decoherence-free subspaces

\section{Introduction}

Continuous-time open quantum random walks (COQRW) on graphs were introduced using the continuous-time limits of open quantum random walks in [19]. It was shown that the limit processes are represented by Quantum Markov Semigroups $(\mathrm{QMSs}) \mathcal{T}=\left(\mathcal{T}_{t}\right)_{t \geq 0}$, i.e., $\mathcal{T}$ is a weakly*-continuous semigroup of completely positive, identity preserving, normal linear maps on the von Neumann algebra $\mathbf{B}(\mathrm{h})$ of all linear bounded operators on a given complex separable

$\begin{array}{lrl}\text { Received: } & \text { May 31, } 2016 \\ \text { Revised: } & \text { August 29, } 2016 \\ \text { Published: } & \text { October 7, } 2016\end{array}$

(C) 2016 Academic Publications, Ltd. url: www.acadpubl.eu 
Hilbert space h. QMSs are a non-commutative extension of Markov semigroups defined in classical probability, they represent an evolution without memory of a microscopic system in accordance with the laws of quantum physics and fit into the framework of open quantum systems (see [12], [13]). The semigroup $\mathcal{T}$ corresponds to the Heisenberg picture in the sense that given any observable $x$, $\mathcal{T}_{t}(x)$ describes its evolution at time $t$. In this way, given a density matrix $\rho$, its dynamics (Schrodinger picture or predual semigroup) is given by the semigroup $\mathcal{T}_{* t}(\rho)$, where $\operatorname{tr}\left(\rho \mathcal{T}_{t}(x)\right)=\operatorname{tr}\left(\mathcal{T}_{* t}(\rho) x\right)$.

Several aspects of temporal evolutions described by COQRWs have been investigated. By example in [2], and [19], relationship with non-Markovian generalized Lindblad master equations is studied. In [6] (in a particular class of COQRWs) convergence to a steady state regardless of the initial state when a graph is connected is showed. In this work we are interested in study decoherence-free subspaces in continuous-time open quantum random walks on graphs.

Decoherence occurs when a quantum system interacts with its environment in an irreversible way. Decoherence and noise ([4, 14, 15, 20, 21, 25] and references therein) typically affect quantum features of a state over its time evolution, however it may be possible to find states with a unitary evolution in some "good" portion of a system.

Two main approaches to decoherence of open quantum systems have been proposed in the literature; both are based on quantum Markov semigroups.

Blanchard and Olkiewicz [3], starting from an algebraic setting, defined environment induced decoherence and found many physical models where the system algebra decomposes as the direct sum of two pieces: a subalgebra, called the decoherence-free algebra, where the semigroup acts homomorphically, a Banach subspace where the semigroup action is purely dissipative (see e.g. papers $[4,5]$ and the references therein) and vanishing as time tends to infinity. The decoherence-free subalgebra was later characterised in $[11,13]$ as the commutant (or generalised commutant for unbounded operators) of certain families of operators arising form the GKSL (Gorini-Kossakowski-Sudarshan-Lindblad) representation of the generator. In particular, decoherence-free subalgebras of COQRWs were studied in [10].

In the approach to decoherence proposed by Lidar et al. [15, 8] registers of a quantum computer are modeled by a quantum open system on a finitedimensional Hilbert space $\mathrm{h}$. The time evolution of states is described by a predual semigroup $\mathcal{T}_{*}$ on the Banach space of trace class operators on $\mathrm{h}$. Given that, for a quantum computer to execute a quantum algorithm efficiently, it needs to evolve by unitary quantum dynamics, in this aprroach, is defined a particular sector where a quantum algorithm is executable: the decoherence- 
free subspaces.

A subspace $h_{f}$ of $\mathrm{h}$ is decoherence-free if the time evolution of states $\omega$ supported in $\mathbf{h}_{\mathbf{f}}$ is given by $\omega \rightarrow e^{-i t K} \omega e^{\mathrm{i} t K}$ for some self-adjoint operator $K$ on $h_{\mathrm{f}}$.

Decoherence-free subspaces were identified in [8] (see also [24]) under some physical (somewhat implicit) assumptions, we refer to [15] for an introduction to the theory of decoherence-free subspaces with a lot of examples and applications to protection of quantum information.

Lidar et al. papers, however, are concerned only with finite-dimensional systems and focus on explicit physical models. Moreover, his method essentially depends on the choice of an orthonormal basis at the outset. This basis is determined by the spectral analysis of the coefficients of the GKSL generator of the quantum Markov semigroup. As a result, this method cannot be extended to infinite dimensions, or to the case of continuous spectra and unbounded coefficients of the GKSL-generator. In [1] they look at the decoherence-free subspace issue from a mathematical point of view and study the following problem: given a quantum Markov semigroup on the algebra B (h) with generator represented in a generalised GKSL form, characterising its decoherence-free subspaces for a possibly infinite dimensional Hilbert space $\mathrm{h}$. We use this approach by study decoherence-free subspaces and temporary changes of quantum correlations in COQRWs.

The structure of the paper is as follows. Section 2 contains the definition of decoherence-free subspaces for any QMSs and some preliminary remarks. In Section 3 we introduce COQRW on graphs and we study his decoherencefree subspace. The temporary change of quantum correlations using geometric quantum discord with bures distance under some assumptions about the semigroup is presented in Section 4.

\section{Decoherence-Free Subspaces}

Fron now on, we denote the von Neumann algebra of all bounded operators on the Hilbert space $h$ by $B(h)$. We recall that an state $\omega$ on $B(h)$ is a positive, trace-one, operator on $\mathrm{h}$, in particular it is a clase trace operator on $\mathrm{h}$. The support $\operatorname{supp}(\omega)$ of $\omega$ is the closed subspace of $\mathrm{h}$ generated by eigenvectors with strictly positive eigenvalues.

Definition 1. A subspace $\mathrm{h}_{\mathrm{f}}$ of $\mathrm{h}$ is called decoherence-free (DF) if there exists a self-adjoint operator $K$ on $h_{\mathbf{f}}$ such that for all state $\omega$ with support in 
$h_{f}$ we have

$$
\mathcal{T}_{* t}(\omega)=e^{-\mathrm{i} t K} \omega e^{\mathrm{i} t K}
$$

for all $t \geq 0$. From now on, we will call that $K$ is associated with $\mathrm{h}_{f}$.

Note that a self-adjoint operator $K$ on $\boldsymbol{h}_{\mathrm{f}}$ can always be extended to the whole Hilbert space $\mathrm{h}$, therefore DF subspaces could be defined in an equivalent way with a self-adjoint operator $K$ on $\mathrm{h}$ leaving the subspace $\mathbf{h}_{\mathbf{f}}$ invariant. In a more precise way, for an unbounded $K$, this means that $e^{-i t K}\left(\mathbf{h}_{\mathbf{f}}\right) \subseteq \mathbf{h}_{\mathbf{f}}$ for all $t \in \mathbb{R}$. Moreover, note that a DF subspace is necessarily closed with respect to the norm topology of $\mathrm{h}$.

Remark. COQRW on graphs are a particular case of predual semigroup of a QMS, where the generator $\mathcal{L}_{*}$ of predual semigroup has a well-known representation. In general, any generator of a QMS is described by the GoriniKossakowski-Sudarshan-Lindblad (GKSL) form

$$
\mathcal{L}_{*}(\rho)=\rho G^{*}+\sum_{s \geq 1} L_{s} \rho L_{s}^{*}+G \rho,
$$

for all state $\rho$ on $\mathrm{B}(\mathrm{h})$, where

$$
G=-\frac{1}{2} \sum_{s \geq 1} L_{s}^{*} L_{s}-i H,
$$

$L_{s}, H \in \mathrm{B}(\mathrm{h})$ wit $H$ selftadjoint, $\left(L_{s}\right)_{s \geq 1}$ is a finite or infinite sequence and the series $\sum_{s \geq 1} L_{s}^{*} L_{s}$ converge strongly .(See [18], theorem 3.16, pag 271.)

Remark. Recall that the operators $L_{\ell}, H \in \mathrm{B}(\mathrm{h})$ in a GKSL representation of $\mathcal{L}$ are not unique, we may, for instance, translate each $L_{\ell}$ by adding multiples $z_{\ell} \mathbb{1}$ of the identity operator $\mathbb{1}$, with $\sum_{\ell}\left|z_{\ell}\right|^{2}<\infty$. In this way we obtain another GKSL representation of $\mathcal{L}$ with $L_{\ell}^{\prime}=L_{\ell}+z_{\ell} \mathbb{1}$ and $H^{\prime}=H+(2 \mathrm{i})^{-1} \sum_{\ell \geq 1}\left(\bar{z}_{\ell} L_{\ell}-\right.$ $\left.z_{\ell} L_{\ell}^{*}\right)$. We refer to [18] p.272-273 for a detailed discussion on this subject.

Using the structure in the generator of semigroup is possible to give necessary and sufficient conditions to find DFs of an arbitrary QMS. This sentence is true when the coefficients of $\mathcal{L}_{*}$ are bounded operator and its also true in the not bounded case. We present the result in the bounded case. (General result is founded in [1], proposition 7 ).

Theorem 2. A subspace $\mathrm{h}_{\mathrm{f}}$ is a DF subspace with associated self-adjoint operator $K$, if and only if in any GKSL representation of $\mathcal{L}$ by means of operators $L_{\ell}, G$ there exist complex numbers $\lambda_{\ell}(\ell \geq 1)$ and a real number $r$ such that $\sum_{\ell \geq 1}\left|\lambda_{\ell}\right|^{2}<\infty$ and 
1. $L_{\ell} u=\lambda_{\ell} u$ for all $u \in \mathrm{h}_{\mathrm{f}}$ and $\ell \geq 1$,

2. $(G+\mathrm{i} K) u=-\left(\frac{1}{2} \sum_{\ell \geq 1}\left|\lambda_{\ell}\right|^{2}+\mathrm{i} r\right) u$ for all $u \in \mathbf{h}_{\mathbf{f}}$.

Proof. Consider the GKSL representation of the generator $\mathcal{L}$ and $h_{\mathrm{f}}$ is a DF subspace with associated operator $K$.

By the well-known polarisation identity (1) also holds for $\omega=|u\rangle\langle v|$ with $u, v \in \mathbf{h}_{\mathbf{f}}$. Differentiating we have

$$
-\mathrm{i}[K,|u\rangle\langle v|]=G|u\rangle\left\langle v\left|+\sum_{\ell}\right| L_{\ell} u\right\rangle\left\langle L_{\ell} v|+| u\right\rangle\langle v| G^{*}
$$

If $v=u$, for all $w \in \mathbf{h}$ orthogonal to $u$ we find

$$
\sum_{\ell}\left|\left\langle w, L_{\ell} u\right\rangle\right|^{2}=0
$$

it follows that $u$ is an eigenvector of all $L_{\ell}$, i.e. $L_{\ell} u=\lambda_{\ell}(u) u$ for $\lambda_{\ell}(u) \in \mathbb{C}$.

The identity (4) for $w \in \mathbf{h}_{\mathrm{f}}$ also yields

$$
\lambda_{\ell}(u)\langle w, u\rangle=\left\langle w, p_{\mathrm{f}} L_{\ell} p_{\mathrm{f}} u\right\rangle=\left\langle p_{\mathrm{f}} L_{\ell}^{*} p_{\mathrm{f}} w, u\right\rangle
$$

i.e. $p_{\mathrm{f}} L_{\ell}^{*} p_{\mathrm{f}} w=0$ if $\langle w, u\rangle=0$ and $p_{\mathrm{f}} L_{\ell}^{*} p_{\mathrm{f}} u=\overline{\lambda_{\ell}}(u) u$ otherwise, showing that the operator $p_{\mathrm{f}} L_{\ell} p_{\mathrm{f}}$ is normal.

We now prove that eigenvalues $\lambda_{\ell}(u)$ do not depend on the choice of the vector $u \in \mathbf{h}_{\mathbf{f}}$. Note, first of all, that eigenvectors $u, v$ in $\mathbf{h}_{\mathbf{f}}$ of $p_{\mathbf{f}} L_{\ell} p_{\mathbf{f}}$ with different eigenvalues $\lambda_{\ell}(u) \neq \lambda_{\ell}(v)$ are orthogonal since

$$
\lambda_{\ell}(v)\langle v, u\rangle=\left\langle p_{\mathrm{f}} L_{\ell}^{*} p_{\mathrm{f}} v, u\right\rangle=\left\langle v, p_{\mathrm{f}} L_{\ell} p_{\mathrm{f}} u\right\rangle=\lambda_{\ell}(u)\langle v, u\rangle .
$$

Since, the Hilbert space $\mathrm{h}$ being separable, the spectrum of $p_{\mathrm{f}} L_{\ell} p_{\mathrm{f}}$ is at most countable, hence totally disconnected. The function on the unit sphere of $h_{f}$

$$
u \rightarrow\left\langle u, L_{\ell} u\right\rangle=\lambda_{\ell}(u)
$$

is continuous and so its range must be connected. It follows that the function $u \rightarrow \lambda_{\ell}(u)$ is constant.

Now, rewriting (3) as

$$
|(G+\mathrm{i} K) u\rangle\langle v|+| u\rangle\left\langle\left.(G+\mathrm{i} K) v\left|+\sum_{\ell}\right| \lambda_{\ell}\right|^{2} \mid u\right\rangle\langle v|=0,
$$


we see that $u$ and $v$ are also eigenvectors for $G+\mathrm{i} K$. The eigenvalues $z(u)$ and $z(v)$ fulfill the identity

$$
\left(z(u)+\overline{z(v)}+\sum_{\ell}\left|\lambda_{\ell}\right|^{2}\right)|u\rangle\langle v|=0,
$$

hence $z(u)+\overline{z(v)}+\sum_{\ell}\left|\lambda_{\ell}\right|^{2}=0$ for all $u, v \in \mathbf{h}_{\mathbf{f}}$. Taking $u=v$ we see that

$$
z(u)=-\mathrm{i} r(u)-\frac{1}{2} \sum_{\ell}\left|\lambda_{\ell}\right|^{2}
$$

for some $r(u) \in \mathbb{R}$. Finally, replacing this in (6), we see that $r(u)$ must be independent of $u \in \mathbf{h}_{\mathrm{f}}$.

Conversely, suppose that 1 . and 2. hold, then we compute immediately

$$
-\mathrm{i}[K,|u\rangle\langle v|]=\mathcal{L}_{*}(|u\rangle\langle v|)
$$

for all $u, v \in \mathbf{h}_{\mathbf{f}}$. Since $\mathbf{h}_{\mathbf{f}}$ is $K$-invariant, replacing $u, v$ by

$$
e^{-\mathrm{i}(t-s) K} u, e^{-\mathrm{i}(t-s) K} v \in \mathbf{h}_{\mathbf{f}}
$$

the above relationship also holds for $\left|e^{-\mathrm{i}(t-s) K} u\right\rangle\left\langle e^{-\mathrm{i}(t-s) K} v\right|$ and we have,

$$
\begin{aligned}
& \frac{d}{d s} \mathcal{T}_{* s}\left(e^{-\mathrm{i}(t-s) K}|u\rangle\langle v| e^{\mathrm{i}(t-s) K}\right) \\
& =\mathcal{T}_{* s}\left(\left(\mathcal{L}_{*}+\mathrm{i}[K, \cdot]\right)\left(e^{-\mathrm{i}(t-s) K}|u\rangle\langle v| e^{\mathrm{i}(t-s) K}\right)\right. \\
& =0 .
\end{aligned}
$$

Therefore

$$
\mathcal{T}_{* t}(|u\rangle\langle v|)=e^{-\mathrm{i} t K}|u\rangle\langle v| e^{\mathrm{i} t K}
$$

and $h_{f}$ is decoherence-free.

Remark. The above result shows that, translating the operators $L_{\ell}$ by $-\lambda_{\ell}$, we find another GKSL representation of $\mathcal{L}$ with $L_{\ell}^{\prime}=L_{\ell}-\lambda_{\ell} \mathbb{1}$ and $H^{\prime}=$ $H+(2 \mathrm{i})^{-1} \sum_{\ell}\left(\bar{z}_{\ell} L_{\ell}-z_{\ell} L_{\ell}^{*}\right)$. In this way, since $\sum_{\ell>1}\left(L_{\ell}^{\prime}\right)^{*} L_{\ell}^{\prime}$ vanishes on $\mathbf{h}_{\mathbf{f}}$, we find as self-adjoint operator $K$ associated with $\mathrm{h}_{\mathrm{f}}$ the generator of the oneparameter group originating from the action of the semigroup in the new GKSL representation of $\mathcal{L}$.

This theorem provides a recipe for finding DF subspaces. First of all look for common eigenspaces for all the operators $L_{\ell}$, then, translate $L_{\ell}$ to $L_{\ell}-\lambda_{\ell} \mathbb{1}$ 
with eigenvalues $\lambda_{\ell}$ finding a new GKSL representation of the generator $\mathcal{L}$. The intersection of common eigenspaces of all the operators $L_{\ell}$ is now the common kernel of all the operators $L_{\ell}-\lambda_{\ell} \mathbb{1}$. Finally check that the operator $G$ founded in the new GSKL representation of $\mathcal{L}$ leaves the common kernel invariant and is anti self-adjoint on this subspace.

Remark. A $K$-invariant subspace of a DF subspace is itself a DF subspace, therefore we will be interested in maximal DF subspaces.

\section{COQRWs: Some Properties and Associated Decoherence-Free Subspaces}

In this section we describe the model, some properties and DFs of COQRWs.

Let $\mathcal{G}$ be a graph such that the set of vertexes is given by $\mathcal{V}=\{1, \ldots, d\}$, with $d \geq 2$. To each vertex $j \in \mathcal{V}$ we associate an element $e_{j}$ in the canonical basis of $\mathbb{C}^{d}$, moreover, all vertex in $\mathcal{V}$ have degrees of freedom given, in this article, by the simplest space $\mathbb{C}^{2}$ equipped with an orthonormal basis $\left(f_{j}\right)_{j=1,2}$, therefore the dynamic is describe by operators in $\mathrm{h}=\mathbb{C}^{2} \otimes \mathbb{C}^{d}$ in the following way: for all $j, m \in \mathcal{V}$ such that $j \neq m$, we fix $B_{m j} \in M_{2}(\mathbb{C})$ standing for the effect of passing from the vertex $m$ to the vertex $j$. We also define the sum of all the effects when one leaves the vertex $m$

$$
B_{m m}^{*} B_{m m}:=\sum_{j \neq m} B_{m j}^{*} B_{m j} .
$$

\section{Proposition 3.}

$$
\operatorname{Ker}\left(B_{m m}\right)=\bigcap_{j \neq m} \operatorname{Ker}\left(B_{m j}\right)
$$

for all $m \in \mathcal{V}$.

Proof. $x \in \operatorname{Ker}\left(B_{m m}\right)$ if and only if $B_{m m} x=0$ if and only if $x \in$ $\operatorname{Ker}\left(B_{m m}^{*} B_{m m}\right)$ if and only if

$$
\sum_{j \neq m} B_{m j}^{*} B_{m j} x=0
$$

if and only if $\sum_{j \neq m}\left\|B_{m j} x\right\|^{2}=\sum_{j \neq m}\left\langle B_{m j} x, B_{m j} x\right\rangle=0$ if and only if $B_{m j} x=0$ for all $j \neq m$ if and only if $x \in \bigcap_{j \neq m} \operatorname{Ker}\left(B_{m j}\right)$. 
The continuous-time open quantum random walk (COQRW) is the predual semigroup $\mathcal{T}_{*}=\left(\mathcal{T}_{* t}\right)_{t \geq 0}$ acting on the set of class-trace operator $\mathcal{I}(\mathrm{h})$ defined in $\mathrm{h}=\mathbb{C}^{2} \otimes \mathbb{C}^{d}$, its Lindblad generator is given by

$$
\mathcal{L}_{*}(\rho)=\rho G^{*}+\sum_{j, m \in \mathcal{V} ; j \neq m} L_{m j} \rho L_{m j}^{*}+G \rho,
$$

for all $\rho \in \mathcal{I}(\mathrm{h})$, where

$$
\begin{gathered}
L_{m j}=B_{m j} \otimes\left|e_{j}\right\rangle\left\langle e_{m}\right|, \quad j \neq m \\
G=-i H-\frac{1}{2} \sum_{j \neq m} L_{m j}^{*} L_{m j}=-i H-\frac{1}{2} \sum_{m=1}^{k} B_{m m}^{*} B_{m m} \otimes\left|e_{m}\right\rangle\left\langle e_{m}\right|
\end{gathered}
$$

with $H=H^{*}$ bounded operator in $\mathrm{h}$.

Remark. Let $B: \mathbb{C}^{2} \rightarrow \operatorname{Rang}(B), P: \mathbb{C}^{d} \rightarrow \operatorname{Rang}(P)$ be linear operators then

$$
\operatorname{Ker}(B \otimes P)=\left(K \operatorname{Ker}(B) \otimes \mathbb{C}^{d}\right) \oplus\left(\mathbb{C}^{2} \otimes \operatorname{Ker}(P)\right)
$$

(see theorem 2.19 in [9]).

We denote $\left\{e_{m}\right\}^{\perp}$ to the orthogonal subespace of subspace generated by $e_{m}$.

\section{Proposition 4.}

$$
\begin{aligned}
& \bigcap_{j \in \mathcal{V}}\left[\left(\operatorname{Ker}\left(B_{m j}\right) \otimes \mathbb{C}^{d}\right) \oplus\left(\mathbb{C}^{2} \otimes\left\{e_{m}\right\}^{\perp}\right)\right]= \\
& \left(\bigcap_{j \in \mathcal{V}} \operatorname{Ker}\left(B_{m j}\right) \otimes \mathbb{C}^{d} \oplus\left(\mathbb{C}^{2} \otimes\left\{e_{m}\right\}^{\perp}\right)\right.
\end{aligned}
$$

for all $m \in \mathcal{V}$.

Proof. We take

$$
\Delta_{1}:=\bigcap_{j \in \mathcal{V}}\left[\left(\operatorname{Ker}\left(B_{m j}\right) \otimes \mathbb{C}^{d}\right) \oplus\left(\mathbb{C}^{2} \otimes\left\{e_{m}\right\}^{\perp}\right)\right]
$$

and

$$
\Delta_{2}:=\left(\bigcap_{j \in \mathcal{V}} \operatorname{Ker}\left(B_{m j}\right) \otimes \mathbb{C}^{d} \oplus\left(\mathbb{C}^{2} \otimes\left\{e_{m}\right\}^{\perp}\right) .\right.
$$


Given $m \in \mathcal{V}$, its clear that $\Delta_{2} \subset\left(\operatorname{Ker}\left(B_{m j}\right) \otimes \mathbb{C}^{d}\right) \oplus\left(\mathbb{C}^{2} \otimes\left\{e_{m}\right\}^{\perp}\right)$ for all $j \in \mathcal{V}$ then $\Delta_{2} \subset \Delta_{1}$.

Moreover, $\Delta_{1} \subset\left(\operatorname{Ker}\left(B_{m j}\right) \otimes \mathbb{C}^{d}\right) \oplus\left(\mathbb{C}^{2} \otimes\left\{e_{m}\right\}^{\perp}\right)$ for all $j \in \mathcal{V}$ then $\Delta_{1} \subset \Delta_{2}$.

A description of decoherence-free subspace for COQRW is obtained by applying Theorem 2, Proposition 3, Proposition 4, and equality (9).

Theorem 5. Let $\mathrm{h}_{f}$ be DF subspace of COQRW and

$$
\mathcal{V}_{0}:=\left\{i \in \mathcal{V} ; B_{i j}=0 \quad \forall j \neq i\right\}
$$

then

$$
\mathrm{h}_{f}=\bigcap_{m \in \mathcal{V}-\mathcal{V}_{0}}\left[\left(\operatorname{Ker}\left(B_{m m}\right) \otimes \mathbb{C}^{d}\right) \oplus\left(\mathbb{C}^{2} \otimes\left\{e_{m}\right\}^{\perp}\right)\right]
$$

with $K=H$ associated self-adjoint operator.

Proof. First note that the only eigenvalue of an operator

$$
L_{m j}=B_{m j} \otimes\left|e_{j}\right\rangle\left\langle e_{m}\right|
$$

with $m \neq j$ and $B_{m j} \neq 0$ is 0 . Indeed, if we suppose that exist $u=\sum_{k, s} u_{k s} f_{k} \otimes$ $e_{s}$ eigenvector of $L_{m j}$ with eigenvalue associated $\lambda \neq 0$ then

$$
\begin{aligned}
\lambda \sum_{k, s} u_{k s} f_{k} \otimes e_{s} & =\lambda u=L_{m j} u=\sum_{k, s} u_{k s} B_{m j} f_{k} \otimes\left|e_{j}\right\rangle\left\langle e_{m}\right| e_{s} \\
& =\sum_{k} u_{k m} B_{m j} f_{k} \otimes e_{j}
\end{aligned}
$$

so $\lambda \sum_{k, s} u_{k s}\left\langle f_{k} \otimes e_{s}, f_{r} \otimes e_{m}\right\rangle=0$ for all $m \neq j$, i.e., $\lambda u_{r m}=0$ for all $r$ and $m \neq j$. Thus $u=\sum_{k} u_{k j} f_{k} \otimes e_{j}$ hence

$$
\lambda u=\lambda \sum_{k} u_{k j} f_{k} \otimes e_{j}=L_{m j} u=\sum_{k} u_{k j} B_{m j} f_{k} \otimes\left|e_{j}\right\rangle\left\langle e_{m}\right| e_{j}=0
$$

then $u=0$ (we suppose $\lambda \neq 0$ ) and by other hand, if $u$ is eigenvector then $u \neq 0$, this is a contradiction. Therefore $\lambda=0$.

Second,by (9) note that

$$
\bigcap_{\substack{m, j \in \mathcal{V} \\ m \neq j}} \operatorname{Ker}\left(L_{m j}\right)=\bigcap_{\substack{m \in \mathcal{V}-\mathcal{V}_{0} \\ j \in \mathcal{V}}} \operatorname{Ker}\left(L_{m j}\right)=\bigcap_{\substack{m \in \mathcal{V}-\mathcal{V}_{0} \\ j \in \mathcal{V}}} \operatorname{Ker}\left(B_{m j} \otimes\left|e_{j}\right\rangle\left\langle e_{m}\right|\right)
$$




$$
\begin{aligned}
& =\bigcap_{\substack{m \in \mathcal{V}-\mathcal{V}_{0} \\
j \in \mathcal{V}}}\left(\operatorname{Ker}\left(B_{m j}\right) \otimes \mathbb{C}^{d}\right) \oplus\left(\mathbb{C}^{2} \otimes \operatorname{Ker}\left(\left|e_{j}\right\rangle\left\langle e_{m}\right|\right)\right. \\
& =\bigcap_{\substack{m \in \mathcal{V}-\mathcal{V}_{0} \\
j \in \mathcal{V}}}\left[\left(\operatorname{Ker}\left(B_{m j}\right) \otimes \mathbb{C}^{d}\right) \oplus\left(\mathbb{C}^{2} \otimes\left\{e_{m}\right\}^{\perp}\right)\right] .
\end{aligned}
$$

Finally, we see that if $u \in \bigcap_{\substack{m, j \in \mathcal{V} \\ m \neq j}} \operatorname{Ker}\left(L_{m j}\right)$ then $L_{m j}^{*} L_{m j} u=0$ for all $m \neq j$. Taking $K=H$ we otain

$$
(G+i K) u=i(-H+K) u=0 .
$$

Applying Theorem 2 with $K=H$ we obtain that .

$$
\mathrm{h}_{f}=\bigcap_{\substack{m \in \mathcal{V}-\mathcal{V}_{0} \\ j \in \mathcal{V}}}\left[\left(\operatorname{Ker}\left(B_{m j}\right) \otimes \mathbb{C}^{d}\right) \oplus\left(\mathbb{C}^{2} \otimes\left\{e_{m}\right\}^{\perp}\right)\right]
$$

Using Propositions 3 and 4 it follows that

$$
\begin{aligned}
\mathrm{h}_{f} & =\bigcap_{\substack{m \in \mathcal{V}-\mathcal{V}_{0} \\
j \in \mathcal{V}}}\left[\left(\operatorname{Ker}\left(B_{m j}\right) \otimes \mathbb{C}^{d}\right) \oplus\left(\mathbb{C}^{2} \otimes\left\{e_{m}\right\}^{\perp}\right)\right] \\
& =\bigcap_{m \in \mathcal{V}-\mathcal{V}_{0}} \bigcap_{j \in \mathcal{V}}\left[\left(\operatorname{Ker}\left(B_{m j}\right) \otimes \mathbb{C}^{d}\right) \oplus\left(\mathbb{C}^{2} \otimes\left\{e_{m}\right\}^{\perp}\right)\right] \\
& =\bigcap_{m \in \mathcal{V}-\mathcal{V}_{0}}\left[\left(\bigcap_{j \in \mathcal{V}} \operatorname{Ker}\left(B_{m j}\right) \otimes \mathbb{C}^{d}\right) \oplus\left(\mathbb{C}^{2} \otimes\left\{e_{m}\right\}^{\perp}\right)\right] \\
& =\bigcap_{m \in \mathcal{V}-\mathcal{V}_{0}}\left(\operatorname{Ker}\left(B_{m m}\right) \otimes \mathbb{C}^{d}\right) \oplus\left(\mathbb{C}^{2} \otimes\left\{e_{m}\right\}^{\perp}\right)
\end{aligned}
$$

Remark. Theorem 5 establish the sectors, where a quantum algorithm describe by COQRWs on graphs is executable.

Hereinafter, we study some properties of COQRWs when $H=0$, in this case, we obtain that

$$
G=-\frac{1}{2} \sum_{j \neq m} L_{m j}^{*} L_{m j}=-\frac{1}{2} \sum_{m=1}^{k} B_{m m}^{*} B_{m m} \otimes\left|e_{m}\right\rangle\left\langle e_{m}\right|
$$


Writing any $\rho$ in $\mathrm{h}=\mathbb{C}^{2} \otimes \mathbb{C}^{\mathcal{V}}$ as a block matrix with respect to the canonical basis of $\mathbf{B}\left(\mathbb{C}^{\mathcal{V}}\right)$ then we obtain

$$
\rho=\sum_{p, q \in \mathcal{V}} \widetilde{\rho}_{p q} \otimes\left|e_{q}\right\rangle\left\langle e_{p}\right|, \quad \widetilde{\rho}_{p q} \in M_{2}(\mathbb{C}) .
$$

We call diagonal subspace, and denote it by $\mathcal{D}$ the subspace generated by the elements $X_{k k} \otimes\left|e_{k}\right\rangle\left\langle e_{k}\right|$ with $X_{k k} \in M_{2}(\mathbb{C})$. Let $\mathcal{E}: \mathrm{B}(\mathrm{h}) \rightarrow \mathcal{D}$ be the conditional expectation with range $\mathcal{D}$ defined by

$$
\mathcal{E}(x)=\sum_{k} X_{k k} \otimes\left|e_{k}\right\rangle\left\langle e_{k}\right|
$$

and let $\mathcal{E}_{*}$ be the predual map on trace class operators with range

$$
\mathcal{E}_{*}(\rho)=\sum_{k \in \mathcal{V}} \widetilde{\rho}_{k k} \otimes\left|e_{k}\right\rangle\left\langle e_{k}\right|
$$

i.e., $\mathcal{E}_{*}: \mathcal{I}(\mathrm{h}) \rightarrow \mathcal{D}_{*}$ where

$$
\mathcal{D}_{*}:=\left\{\sigma \in \mathcal{I}(\mathrm{h}) ; \sigma=\sum_{k \in \mathcal{V}} \widetilde{\sigma}_{k k} \otimes\left|e_{k}\right\rangle\left\langle e_{k}\right|, \widetilde{\sigma}_{k k} \in M_{2}(\mathbb{C})\right\} .
$$

By equality (10) and positivity of $\sigma$, it follows the positivity of $\widetilde{\sigma}_{p p} \in M_{2}(\mathbb{C})$ for all $p \in \mathcal{V}$. Moreover, $\operatorname{tr}(\sigma)=1$ if and only if $\sum_{p} \operatorname{tr}\left(\widetilde{\sigma}_{p p}\right)=1$, then

$$
\sigma=\sum_{p \in \mathcal{V}} c_{p} \sigma_{p p} \otimes\left|e_{p}\right\rangle\left\langle e_{p}\left|+\sum_{p \neq q} \sigma_{p q} \otimes\right| e_{q}\right\rangle\left\langle e_{p}\right|
$$

with $c_{p}:=\operatorname{tr}\left(\widetilde{\sigma}_{p p}\right) \geq 0$ for all $p \in \mathcal{V}, \quad \sum_{p} c_{p}=1, \quad \sigma_{p p}:=\frac{1}{c_{p}} \widetilde{\sigma}_{p p}\left(\right.$ if $\left.c_{p}>0\right)$ states in $\mathbb{C}^{2}$ and $\sigma_{p q}:=\widetilde{\sigma}_{p q} \in M_{2}(\mathbb{C})$ for all $p \neq q$.

Therefore

$$
\mathcal{D}_{*}=\left\{\sigma \in \mathcal{I}(\mathrm{h}) ; \sigma=\sum_{p \in \mathcal{V}} c_{p} \sigma_{p p} \otimes\left|e_{p}\right\rangle\left\langle e_{p}\right|, \sum_{p} c_{p}=1, \sigma_{p p} \in \mathcal{I}\left(\mathbb{C}^{2}\right)\right\}
$$

and $\mathcal{I}(\mathrm{h})=\mathcal{D}_{*} \oplus \mathcal{D}_{* o f f}$ with $\mathcal{D}_{* o f f}=\mathcal{E}_{*}^{\perp}(\mathcal{I}(\mathrm{h})), \mathcal{E}_{*}^{\perp}:=I-\mathcal{E}_{*}$.

Additionally, we obtain that if COQRWs has null-Hamiltonian, that is, his generator is given by (8) with $H=0$ then

$$
\mathcal{L}_{*}(\rho)=-\frac{1}{2} \sum_{j \neq m}\left(B_{j j}^{*} B_{j j} \rho_{j m}+\rho_{j m} B_{m m}^{*} B_{m m}\right) \otimes\left|e_{j}\right\rangle\left\langle e_{m}\right|
$$




$$
\begin{aligned}
& -\frac{1}{2} \sum_{m} c_{m}\left(B_{m m}^{*} B_{m m} \rho_{m m}+\rho_{m m} B_{m m}^{*} B_{m m}\right) \otimes\left|e_{m}\right\rangle\left\langle e_{m}\right| \\
& +\sum_{m} \sum_{j \neq m} c_{j} B_{j m} \rho_{j j} B_{j m}^{*} \otimes\left|e_{m}\right\rangle\left\langle e_{m}\right|
\end{aligned}
$$

hence $\mathcal{L}_{*}\left(\mathcal{D}_{*}\right) \subset \mathcal{D}_{*}$ y $\mathcal{L}_{*}\left(\mathcal{D}_{* o f f}\right) \subset \mathcal{D}_{* o f f}$ then $\mathcal{T}_{* t}\left(\mathcal{D}_{*}\right) \subset \mathcal{D}_{*}$ y $\mathcal{T}_{* t}\left(\mathcal{D}_{* o f f}\right) \subset$ $\mathcal{D}_{* o f f}$ for all $t \geq 0$, i.e., $\mathcal{D}_{*}$ and $\mathcal{D}_{* o f f}$ are $\mathcal{T}_{*}$-invariants, equivalently

$$
\mathcal{T}_{* t} \circ \mathcal{E}_{*}=\mathcal{E}_{*} \circ \mathcal{T}_{* t}, \quad \mathcal{T}_{* t} \circ \mathcal{E}_{*}^{\perp}=\mathcal{E}_{*}^{\perp} \circ \mathcal{T}_{* t}
$$

for all $t \geq 0$.

\section{Temporary Change in Quantum Correlations}

In this section we measure temporary changes in quantum correlations using geometric quantum discord with Bures distance when COQRWs has nullHamiltonian, that is, his generator is given by (8) with $H=0$. In particular, we characterize the decay of correlations to zero, showing that turns out to be closely related with the structure of decoherence-free subspaces.

The Bures distances between states $\rho_{1}, \rho_{2} \in \mathcal{I}(\mathrm{h})$ with $\mathrm{h}=\mathbb{C}^{2} \otimes \mathbb{C}^{d}$ is given by $d_{B}\left(\rho_{1}, \rho_{2}\right)=\left[2\left(1-\sqrt{F\left(\rho_{1}, \rho_{2}\right)}\right)\right]^{1 / 2}$, where $F\left(\rho_{1}, \rho_{2}\right)$ is the fidelity between $\rho_{1}$ and $\rho_{2}, F\left(\rho_{1}, \rho_{2}\right)=\left[\operatorname{tr}\left(\left[\sqrt{\rho_{2}} \rho_{1} \sqrt{\rho_{2}}\right]^{1 / 2}\right)\right]^{2}$ and geometric quantum discord with Bures distance of a state $\rho \in \mathcal{I}(\mathrm{h})$ has been defined as

$$
D_{\mathcal{D}_{*}}(\rho):=d_{B}\left(\rho, \mathcal{D}_{*}\right)^{2}=\min _{\sigma \in \mathcal{D}_{*}} d_{B}(\rho, \sigma)^{2}=2\left(1-\sqrt{F_{\mathcal{D}_{*}}(\rho)}\right)
$$

with $F_{\mathcal{D}_{*}}(\rho):=\max _{\sigma \in \mathcal{D}_{*}} F(\rho, \sigma)$ (see [16],[17],[22],[23]).

The Bures distance can be used to bound from below and above the trace distance $d_{1}(\rho, \sigma)=\operatorname{tr}(|\rho-\sigma|)$ (see [7]) as follows:

$$
d_{B}(\rho, \sigma)^{2} \leq d_{1}(\rho, \sigma) \leq\left[1-\left(1-\frac{1}{2} d_{B}(\rho, \sigma)^{2}\right)^{2}\right]^{1 / 2}
$$

Theorem 6. Let $\mathcal{T}$ be a COQRW with null-Hamiltonian then the following assertions are equivalent: 
(a)

$$
\mathrm{h}_{f}=\bigcap_{m \in \mathcal{V}-\mathcal{V}_{0}}\left[\left(\operatorname{Ker}\left(B_{m m}\right) \otimes \mathbb{C}^{d}\right) \oplus\left(\mathbb{C}^{2} \otimes\left\{e_{m}\right\}^{\perp}\right)\right]
$$

with at most a unique $m \in \mathcal{V}-\mathcal{V}_{0}$ such that $\operatorname{Ker}\left(B_{m m}\right) \neq 0$.

(b) $\lim _{t \rightarrow 0} D_{\mathcal{D}_{*}}\left(\mathcal{T}_{* t}(\rho)\right)=0$ for all state $\rho$.

(c) $\left\{\rho ; \operatorname{supp}(\rho) \subset \mathrm{h}_{\mathbf{f}}\right\} \subset\left\{\rho ; \mathcal{T}_{* t}(\rho)=\rho\right.$, for all $\left.t \geq 0\right\} \subset \mathcal{D}_{*}$.

Proof. First note that $B_{m m}^{*} B_{m m}$ are positive-semidefinite matrices then there exist unitary matrices $U_{m}$ such that

$$
B_{m m}^{*} B_{m m}=U_{m} D_{m} U_{m}^{*}
$$

with $D_{m}$ diagonal matrix where his elements $\left(d_{m}^{i}\right)_{i=1,2}$ are non-negative.

Let $m \neq j$, with $m, j \in \mathcal{V}, \varrho \otimes\left|e_{m}\right\rangle\left\langle e_{j}\right| \in \mathcal{D}_{* o f f}$. Using equation (14) we obtain that

$$
\begin{aligned}
\mathcal{L}_{*}\left(\varrho \otimes\left|e_{m}\right\rangle\left\langle e_{j}\right|\right) & =-\frac{1}{2}\left(B_{m m}^{*} B_{m m} \varrho+\varrho B_{j j}^{*} B_{j j}\right) \otimes\left|e_{m}\right\rangle\left\langle e_{j}\right| \\
& =-\frac{1}{2}\left(U_{m} D_{m} U_{m}^{*} \varrho+\varrho U_{j} D_{j} U_{j}^{*}\right) \otimes\left|e_{m}\right\rangle\left\langle e_{j}\right| .
\end{aligned}
$$

In particular, taking $\varrho=U_{m} Z U_{j}^{*}$ for an arbitrary operator $Z=\left(Z_{r s}\right)_{r, s=1,2} \in$ $M_{2}(\mathbb{C})$, we obtain

$$
\begin{aligned}
\mathcal{L}_{*}\left(\varrho \otimes\left|e_{m}\right\rangle\left\langle e_{j}\right|\right) & =-\frac{1}{2}\left(U_{m} D_{m} Z U_{j}^{*}+U_{m} Z D_{j} U_{j}^{*}\right) \otimes\left|e_{m}\right\rangle\left\langle e_{j}\right| \\
& =U_{m}\left[-\frac{1}{2}\left(D_{m} Z+Z D_{j}\right)\right] U_{j}^{*} \otimes\left|e_{m}\right\rangle\left\langle e_{j}\right|
\end{aligned}
$$

and recursively, we get

$$
\mathcal{L}_{*}^{n}\left(\varrho \otimes\left|e_{m}\right\rangle\left\langle e_{j}\right|\right)=U_{m} \delta_{j m}^{(n)}(Z) U_{j}^{*} \otimes\left|e_{m}\right\rangle\left\langle e_{j}\right| \quad \text { for all } n \in \mathbb{N},
$$

where $\delta_{j m}(Z):=-\frac{1}{2}\left(D_{m} Z+Z D_{j}\right)$ and $\delta_{j m}^{(n)}=\underbrace{\delta_{j m} \circ \delta_{j m} \circ \ldots \circ \delta_{j m}}$. Hence

$$
\mathcal{T}_{* t}\left(\varrho \otimes\left|e_{m}\right\rangle\left\langle e_{j}\right|\right)=U_{m} e^{t \delta_{j m}}\left(U_{m}^{*} \varrho U_{j}\right) U_{j}^{*} \otimes^{n}\left|e_{m}^{\text {times }}\right\rangle\left\langle e_{j}\right| .
$$

Since $\delta_{j m}(Z)=-\frac{1}{2}\left(D_{m} Z+Z D_{j}\right)=\left(-1 / 2\left(d_{m}^{p}+d_{j}^{q}\right) Z_{p q}\right)_{p q}$ then $\delta_{j m}^{(n)}(Z)=$ $\left((-1 / 2)^{n}\left(d_{m}^{p}+d_{j}^{q}\right)^{n} Z_{p q}\right)_{p q}$ and therefore

$$
e^{t \delta_{j m}}(Z)=\left(\exp \left[(-1 / 2) t\left(d_{m}^{p}+d_{j}^{q}\right)\right] Z_{p q}\right)_{p q} \quad \text { for all } t \geq 0
$$


By (4) and (20), we obtain that

$$
\mathcal{T}_{* t}\left(\varrho \otimes\left|e_{m}\right\rangle\left\langle e_{j}\right|\right)=U_{m}\left[\left(e^{-\frac{1}{2} t\left(d_{m}^{p}+d_{j}^{q}\right)}\left(U_{m}^{*} \varrho U_{j}\right)_{p q}\right)_{p q}\right] U_{j}^{*} \otimes\left|e_{m}\right\rangle\left\langle e_{j}\right|
$$

with $d_{m}^{p} \geq 0, d_{j}^{q} \geq 0$ for all $m, j \in \mathcal{V}, m \neq j$ and $p, q \in\{1,2\}$, for any $\varrho \in M_{2}(\mathbb{C})$ and $t \geq 0$.

If we suppose that

$$
\mathrm{h}_{f}=\bigcap_{m \in \mathcal{V}-\mathcal{V}_{0}}\left[\left(\operatorname{Ker}\left(B_{m m}\right) \otimes \mathbb{C}^{d}\right) \oplus\left(\mathbb{C}^{2} \otimes\left\{e_{m}\right\}^{\perp}\right)\right]
$$

with at most a unique $m \in \mathcal{V}-\mathcal{V}_{0}$ such that $\operatorname{Ker}\left(B_{m m}\right)$ $\neq 0$, then $\operatorname{Ker}\left(B_{m m}^{*} B_{m m}\right) \neq 0$ (by equation (7) and proposition 3) with $B_{m m}^{*} B_{m m} \neq 0\left(m \in \mathcal{V}-\mathcal{V}_{0}\right)$ therefore exists at most a unique $m \in \mathcal{V}$ such that $B_{m m}^{*} B_{m m}$ can have at most one zero eigenvalue, i.e.,exists at most a unique $m \in \mathcal{V}$ and exists at most a unique $p \in\{1,2\}$ such that $d_{m}^{p}=0$ and $d_{j}^{q}>0$ for all $(j, q) \neq(m, p)$. By $(21)$ it follows that

$$
\lim _{t \rightarrow \infty} \mathcal{T}_{* t}\left(\varrho \otimes\left|e_{m}\right\rangle\left\langle e_{j}\right|\right)=0 \quad \text { for any } \varrho \in M_{2}(\mathbb{C}), m, j \in \mathcal{V}, m \neq j,
$$

equivalently

$$
\lim _{t \rightarrow \infty}\left\|\mathcal{T}_{* t}\left(\varrho \otimes\left|e_{m}\right\rangle\left\langle e_{j}\right|\right)\right\|=0 \quad \text { for any } \varrho \in M_{2}(\mathbb{C}), m, j \in \mathcal{V}, m \neq j,
$$

where $\|\cdot\|$ is the operator norm.

Give a arbitrary state

$$
\rho=\sum_{k \in \mathcal{V}} c_{k} \rho_{k k} \otimes\left|e_{k}\right\rangle\left\langle e_{k}\left|+\sum_{k \neq r} \rho_{k r} \otimes\right| e_{r}\right\rangle\left\langle e_{k}\right|,
$$

then

$$
\mathcal{E}^{\perp}(\rho)=\sum_{k \neq r} \rho_{k r} \otimes\left|e_{r}\right\rangle\left\langle e_{k}\right| .
$$

Using (17), (18) and (22), we see that

$$
\begin{aligned}
D_{\mathcal{D}_{*}}\left(\mathcal{T}_{* t}(\rho)\right) & \leq d_{B}\left(\mathcal{T}_{* t}(\rho), \mathcal{E}_{*}\left(\mathcal{T}_{* t}(\rho)\right)\right)^{2} \leq d_{1}\left(\mathcal{T}_{* t}(\rho), \mathcal{E}_{*}\left(\mathcal{T}_{* t}(\rho)\right)\right) \\
& =d_{1}\left(\mathcal{T}_{* t}(\rho), \mathcal{T}_{* t}\left(\mathcal{E}_{*}(\rho)\right)\right)=\operatorname{tr}\left(\mathcal{T}_{* t}\left(\mathcal{E}_{*}^{\perp}(\rho)\right)\right) \\
& =\sum_{k \neq r} \operatorname{tr}\left(\mathcal{T}_{* t}\left(\rho_{k r} \otimes\left|e_{r}\right\rangle\left\langle e_{k}\right|\right)\right)
\end{aligned}
$$




$$
\leq 2 d \sum_{k \neq r}\left\|\mathcal{T}_{* t}\left(\rho_{k r} \otimes\left|e_{r}\right\rangle\left\langle e_{k}\right|\right)\right\| \underset{t \rightarrow \infty}{\longrightarrow} 0
$$

then $(a) \Rightarrow(b)$.

Now, If we suppose that $\lim _{t \rightarrow 0} D_{\mathcal{D}_{*}}\left(\mathcal{T}_{* t}(\rho)\right)=0$ for all state $\rho$. In particular, if we take $\rho$ such that $\mathcal{T}_{* t}(\rho)=\rho$ for all $t \geq 0$ then $D_{\mathcal{D}_{*}}(\rho)=0$, therefore $\rho \in \overline{\mathcal{D}}_{*}^{d}$ where $\overline{\mathcal{D}_{*}} d_{B}$ is the closure of $\mathcal{D}_{*}$ with respect to Bures distance. By inequality (18), it is easy to see $\overline{\mathcal{D}}_{*}^{d}=\mathcal{D}_{*}$ and that

$$
\left\{\rho ; \operatorname{supp}(\rho) \subset \mathrm{h}_{\mathbf{f}}\right\} \subset\left\{\rho ; \mathcal{T}_{* t}(\rho)=\rho \text {, for all } t \geq 0\right\}
$$

therefore $\rho \in \mathcal{D}_{*}$, then $(b) \Rightarrow(c)$.

If $\left\{\rho ; \operatorname{supp}(\rho) \subset \mathbf{h}_{\mathbf{f}}\right\} \subset\left\{\rho ; \mathcal{T}_{* t}(\rho)=\rho, \forall t \geq 0\right\} \subset \mathcal{D}_{*}$ and exists $m, j \in \mathcal{V}-\mathcal{V}_{0}$, $m \neq j$ such that $\operatorname{Ker}\left(B_{m m}\right) \neq 0$ and $\operatorname{Ker}\left(B_{j j}\right) \neq 0$ then exist $d_{m}^{r}$ eigenvalue of $B_{m m}^{*} B_{m m}$ and $d_{j}^{s}$ eigenvalue of $B_{j j}^{*} B_{j j}$ such that $d_{m}^{r}=d_{j}^{s}=0, d_{m}^{l}>0$ if $l \neq r$, and $d_{j}^{k}>0$ if $k \neq s$. Using (21), we see that

$$
\begin{gathered}
\mathcal{T}_{* t}\left(\varrho \otimes\left|e_{m}\right\rangle\left\langle e_{j}\right|\right)=X+Y_{t} \\
X=U_{m}\left[\left(U_{m}^{*} \varrho U_{j}\right)_{r s}\left|f_{r}\right\rangle\left\langle f_{s}\left|+\left(U_{m}^{*} \varrho U_{j}\right)_{s r}\right| f_{s}\right\rangle\left\langle f_{r}\right|\right] U_{j}^{*} \otimes\left|e_{m}\right\rangle\left\langle e_{j}\right|, \\
Y_{t}=U_{m}\left[\left(e^{-\frac{1}{2} t\left(d_{m}^{l}+d_{j}^{k}\right)}\left(U_{m}^{*} \varrho U_{j}\right)_{l k}\right)_{l k}\left|f_{l}\right\rangle\left\langle f_{k}\right|\right. \\
\left.+\left(e^{-\frac{1}{2} t\left(d_{m}^{l}+d_{j}^{k}\right)}\left(U_{m}^{*} \varrho U_{j}\right)_{k l}\right)_{k l}\left|f_{k}\right\rangle\left\langle f_{l}\right|\right] U_{j}^{*} \otimes\left|e_{m}\right\rangle\left\langle e_{j}\right|,
\end{gathered}
$$

where $\mathcal{T}_{* t}(X)=X$ for all $t \geq 0$ and $\lim _{t \rightarrow \infty} Y_{t}=0$. We take $\varrho$ such that

$$
\left(U_{m}^{*} \varrho U_{j}\right)_{r s} \neq 0 \text { and }\left(U_{m}^{*} \varrho U_{j}\right)_{s r} \neq 0
$$

then

$$
\begin{aligned}
X=\lim _{t \rightarrow \infty} \mathcal{T}_{* t}\left(\varrho \otimes\left|e_{m}\right\rangle\left\langle e_{j}\right|\right) & \in \mathcal{D}_{\text {oof* }} \cap\left\{\rho ; \mathcal{T}_{* t}(\rho)=\rho, \forall t \geq 0\right\} \\
& \subset \mathcal{D}_{\text {oof } *} \cap \mathcal{D}_{*}=0 .
\end{aligned}
$$

By (23), we obtain that $\left(U_{m}^{*} \varrho U_{j}\right)_{s r}=\left(U_{m}^{*} \varrho U_{j}\right)_{r s}=0$. This is a contradiction with (24) . Therefore exist at most a unique $m \in \mathcal{V}-\mathcal{V}_{0}$ such that $\operatorname{Ker}\left(B_{m m}\right) \neq$ 0 , then $(c) \Rightarrow(a)$. 


\section{Acknowledgements}

J. Agredo and Universidad Nacional de Colombia want to highlight the financial support from "Patrimonio autónomo fondo nacional de financiamiento para la ciencia, tecnología y la innovación, Francisco Jose de Caldas" and "Universidad Nacional de Colombia (UN)".

\section{References}

[1] J. Agredo, F. Fagnola and R. Rebolledo, Decoherence-free subspaces of a quantum Markov semigroup, J. Math. Phys, 55, (2014),11-32.

[2] A. Barchielli and C. Pellegrini, Jump-difusion unravelling of a non-Markovian generalized Lindblad master equation, J. Math. Phys, 51, (2010), 112104.

[3] Ph. Blanchard, and R. Olkiewicz, Decoherence induced transition from quantum to classical dynamics, Rev. Math. Phys, 15, (2003), 217-243 .

[4] Ph. Blanchard, and R. Olkiewicz, Decoherence as irreversible dynamical process in open quantum systems, Open quantum systems. III, Lectures Notes in Math., 1882, Springer, Berlin, (2006).

[5] R. Carbone, E. Sasso, and V. Umanità, Decoherence for Quantum Markov Semigroups on Matrix Algebras, Annales Henri Poincaré, 14, (2013), 17-37.

[6] L. Chaobin and B. Radhakrishnan, Continuous-time open quantum walks, arXiv:quantph/1604.05652v1,(2016)

[7] L. Chuang and M. Nielsen Quantum Computation and Quantum Information, Cambridge University Press, Cambridge (2000).

[8] I.L. Chuang, D.A. Lidar and K.B. Whaley, Decoherence-free Subspaces for Quantum Computation, Phys. Rev. Lett, 81, (1998), 2594.

[9] K. Conrad, Tensor product II, expository paper, http://www.math.uconn.edu/ kcon$\mathrm{rad} /$ blurbs/

[10] J. Deschamps and V. Umanità, The decoherence-free subalgebra for continuous-time open quantum random walks, preprint, (2014).

[11] A. Dhahri, F. Fagnola and R. Rebolledo, The decoherence-free subalgebra of a quantum Markov semigroup with unbounded generator, Infin. Dimens. Anal. Quantum Probab. Relat, Top., 13, (2010), 413-433.

[12] F. Fagnola, Quantum Markov Semigroups and Quantum Markov Flows, Proyecciones, 18, (1999), 1-144.

[13] F. Fagnola and R. Rebolledo, Algebraic conditions for convergence of a quantum Markov semigroup to a steady state, Infin. Dimens. Anal. Quantum Probab. Relat. Top, 11, (2008), 467-474.

[14] D. Giulini, E. Joos, C. Kiefer, J. Kupsch, I. Stamatescu and H. D. Zeh, Decoherence and the appearance of a classical world in quantum theory, Springer, (1996).

[15] D. Lidar, Review of Decoherence Free Subspaces, Noiseless Subsystems, and Dynamical Decoupling, Adv. Chem. Phys. 154, (2014), 295-354. 
[16] M. Orszag and D. Spehner, Geometric quantum discord with Bures distance, New. Jour. Phys, 15, (2013), 103001.

[17] M. Orszag and D. Spehner, Geometric quantum discord with Bures distance: the qubit case,J. Phys. A: Math. Theor, 47, (2014), 035302.

[18] K.R. Parthasarathy, An Introduction to Quantum Stochastic Calculus, Monographs in Mathematics, Vol. 85, (1992).

[19] C. Pellegrini, Continuous-Time Open Quantum Random Walks and Non-Markovian Lindblad Master Equations, J. Stat. Phys, 154, 3 (2014), 838-865.

[20] R. Rebolledo, Decoherence of quantum Markov semigroups, Ann. Inst. Henri Poincaré (B) Probab. Stat, 41, (2005), 349-373.

[21] R. Rebolledo, A view on decoherence via master equations, Open Sys. Inf. Dyn, 12, (2005), 37-54.

[22] W. Roga, D. Spehner and F. Illuminti, Geometric measures of quantum correlations: characterization, quantification, and comparison by distances and operations, J. Phys. A: Math. Theor, 49,(2016), 235301.

[23] D. Spehner, Quantum correlations and distinguishability of quantum states, J. Math. Phys,55,(2014), 075211.

[24] F. Ticozzi and L. Viola, Quantum Markovian Subsystems: Invariance, Attractivity, and Control, IEEE Trans. Automat Control 53, (2008), 2048.

[25] W.H. Zurek, Decoherence and the Transition from Quantum to Classical, Physics Today, 44, (1991), 36-44. 
